\title{
2010, année du poumon: un modèle suisse pour l'Europe!
}

\section{Werner Karrer ${ }^{a}$, \\ Otto Brändli ${ }^{b}$, \\ Thierry Rochat}

a Dr. med., Chefarzt Luzerner Höhenklinik Montana,

Präsident Schweizerische

Gesellschaft für Pneumologie

b Dr. med., Facharzt für Innere Medizin und Pneumologie, Wald $(\mathrm{ZH})$

c Prof. Dr, Service de Pneumologie, Hôpitaux Universitaires de Genève
Les traductions allemande et française ont été financées par des contributions de la Fondation Swiss Lung (www.swisslung.ch) de QUADRIMED (www.quadrimed.ch), ains que de la Ligue pulmonaire suisse (www.lung.ch).

Le document Air Quality and Health est disponible gratuitement en PDF sur www.ersnet.org/airquality. On peut aussi y accéder par un lien à partir de www.SwissTPH.ch ou à parti du site de la Société Suisse de Pneumologie

www.pneumo.ch

Des copies imprimées au prix de 12 CHF (port compris) peuvent être commandées par e-mail ou fax auprès de: library-sti@unibas.ch, fax: 0612848150 .
A l'occasion de son congrès annuel - le plus grand congrès mondial de Pneumologie et le moment le plus important de l'Année Internationale du Poumon l'European Respiratory Society (ERS) publie une brochure de grande actualité sur le thème: Air Quality and Health. Ce document, destiné aux médecins et aux autres professionnels de la santé présente sur 60 pages l'état des dernières connaissances sur le sujet. Il sera disponible dans de nombreuses langues: français, italien, allemand, catalan, turc, ... La lecture de ce document devrait conduire la communauté médicale lobby par excellence pour la défense de la santé - à mieux faire entendre la voix de la science, plus clairement, plus distinctement et à promouvoir l'action politique. En Europe, la régulation de la qualité de l'air est davantage dominée par la politique que par des préoccupations de qualité et ceci au détriment de la santé. Ainsi les valeurs limites pour les particules fines (PM10 et PM2.5) sont deux fois plus élevées que les recommandations de l'OMS, lesquelles ont cependant été acceptées depuis longtemps par beaucoup de pays.

La brochure de l'ERS est ancrée de trois manières dans la recherche scientifique et dans la politique environnementale de la Suisse. Premièrement, l'ERS a mandaté pour cela une équipe d'auteurs compétents - Prof. Nino Künzli, Dr Laura Perez et Dr Regula Rapp - tous de l'Institut Tropicale et de Santé Publique Suisse (Swiss TPH).

Deuxièmement, la recherche scientifique suisse a contribué de manière décisive à l'état actuel des connaissances dans ce domaine au cours des 20 dernières années. En 1990, avec le projet national de recherche NFP26 intitulé «Homme, Santé, Environnement», le Fonds National Suisse a posé les bases pour un réseau de recherche national transdisciplinaire de premier ordre. La décision de centrer la recherche non pas seulement sur les effets à court terme de la pollution atmosphérique, mais sur ses implications à long terme dans les maladies chroniques était particulièrement visionnaire à l'époque. Un tel objectif nécessite des moyens et des fonds importants, raison pour laquelle peu de pays pouvaient s'attaquer à cette question. Les projets majeurs tels que SCARPOL (avec les enfants) et SAPALDIA (avec les adultes) ont pu ainsi être conduits pendant de nombreuses années. Le projet SAPALDIA (www.SAPALDIA.ch) court déjà depuis 20 ans et se trouve actuellement dans sa troisième phase. Dans la Suisse entière, tous les participants de la première heure au projet sont invités à se rendre dans les centres d'examens pour une enquête approfondie et standardisée (Aarau, Bâle, Davos, Genève,
Lugano, Montana, Payerne et Wald). Les résultats obtenus jusqu'ici par SAPALDIA ont contribué à l'échelle mondiale à orienter la politique de la qualité de l'air. La Suisse appartient aux pays qui grâce à une politique active dans ce domaine ont obtenu de nettes améliorations au cours des vingt dernières années. SAPALDIA a pu démontrer scientifiquement que notre politique de la qualité de l'air a entraîné des effets positifs sur la santé.

\section{II reste à espérer que la commu- nauté médicale européenne se lève en force comme lobby pour la défense de la qualité de l'air}

Ceci nous amène à la troisième dimension helvétique de cette nouvelle brochure publiée par l'ERS: la politique peut être influencée par la recherche scientifique et par la voix de tous ceux qui s'engagent pour la cause de la santé. C'est ici que la communauté médicale joue un rôle important. En 1988 déjà - en avance sur d'autres pays - l'association Médecins en faveur de l'Environnement (www.aefu.ch) s'est engagée activement pour la diffusion des connaissances sur les effets de la pollution de l'air. Ainsi fut publiée en 1988 pour la première fois une brochure «Pollution de l'air et santé» dans les trois langues. Les Médecins en faveur de l'Environnement ont contribué durant toutes ces années au renforcement de la politique suisse de la qualité de l'air. Les progrès importants réalisés en recherche ont conduit l'association à mettre à jour cette synthèse en 1997 (par les Prof. Nino Künzli et Philippe Leuenberger, alors que ce dernier dirigeait l'étude SAPALDIA). Le projet fut officiellement soutenu par la Ligue Pulmonaire Suisse et par les deux sociétés de disciplines médicales de Pneumologie et de Santé Publique, ainsi que par la Confédération.

Ainsi, en 2010, cette «idée suisse» fait un tabac en Europe. Il reste à espérer que la communauté médicale européenne se lève en force comme lobby pour la défense de la qualité de l'air, afin que les possibilités d'amélioration soient appliquées de manière décisive dans les zones fortement touchées, en particulier du sud et de l'est du continent. La Suisse en profitera. Sans amélioration à l'échelle européenne il sera difficile à notre pays de faire se rejoindre qualité de l'air et protection de la santé, car une partie de la charge toxique est bel et bien importée par les airs. 